GA-A23992

\title{
TRANSPORT OF ELM ENERGY AND PARTICLES INTO THE SOL AND DIVERTOR OF DIII-D
}

\author{
by \\ A.W. LEONARD, J.A. BOEDO, M.E. FENSTERMACHER, R.J. GROEBNER, \\ M. GROTH, C.J. LASNIER, M.A. MAHDAVI, T.H. OSBORNE, \\ D.L. RUDAKOV, T.W. PETRIE, and J.G. WATKINS
}




\section{DISCLAIMER}

This report was prepared as an account of work sponsored by an agency of the United States Government. Neither the United States Government nor any agency thereof, nor any of their employees, makes any warranty, express or implied, or assumes any legal liability or responsibility for the accuracy, completeness, or usefulness of any information, apparatus, product, or process disclosed, or represents that its use would not infringe privately owned rights. Reference herein to any specific commercial product, process, or service by trade name, trademark, manufacturer, or otherwise, does not necessarily constitute or imply its endorsement, recommendation, or favoring by the United States Government or any agency thereof. The views and opinions of authors expressed herein do not necessarily state or reflect those of the United States Government or any agency thereof. 


\title{
TRANSPORT OF ELM ENERGY AND PARTICLES INTO THE SOL AND DIVERTOR OF DIII-D
}

by

A.W. LEONARD, J.A. BOEDO, † M.E. FENSTERMACHER, ${ }^{\ddagger}$ R.J. GROEBNER, M. GROTH, C.J. LASNIER, M.A. MAHDAVI, T.H. OSBORNE, D.L. RUDAKOV, ${ }^{\dagger}$ T.W. PETRIE, and J.G. WATKINS $\diamond$

\author{
†University of California, San Diego \\ ‡Lawrence Livermore National Laboratory \\ $\checkmark$ Sandia National Laboratories
}

This is a preprint of a paper presented at the 15th International Conference on Plasma Surface Interactions in Controlled Fusion Devices, May 27-31, 2002, in Gifu, Japan, and to be published in the Proceedings.

\section{Work supported by the U.S. Department of Energy} under Contracts DE-AC03-99ER54463 W-7405-ENG-48, DE-AC04-94AL84000, and Grant DE-FG03-95ER54294 


\begin{abstract}
We report on DIII-D data that reveal the underlying processes responsible for transport of energy and particles from the edge pedestal to the divertor target during edge-localized modes (ELMs). The separate convective and conductive transport of energy due to an ELM is determined by Thomson scattering measurements of electron density and temperature in the pedestal. Conductive transport is measured as a drop in pedestal temperature and decreases with increasing density. The convective transport of energy, measured as a loss of density from the pedestal, however, remains constant as a function of density. From the SOL ELM energy is quickly carried to the divertor target. An expected sheath limit to the ELM heat flux set by the slower arrival of pedestal ions is overcome by additional ionization of neutrals generated from the divertor target as evidenced by a fast, $\sim 100 \mu \mathrm{s}$, rise in divertor density. A large in/out asymmetry of the divertor ELM heat flux is observed at high density, but becomes nearly symmetric at low density.
\end{abstract}




\section{INTRODUCTION}

The divertor target heat flux due to edge-localized-modes (ELMs) is a well recognized concern for the next generation of burning plasma tokamak experiments such as ITERFEAT $[1,2]$. While a robust $\mathrm{H}$-mode edge pedestal is needed for adequate confinement in a future burning plasma, steady state $\mathrm{H}$-mode operation typically requires periodic relaxation of the edge barrier through ELMs. The transient energy and particles released from the pedestal into the SOL due to ELMs can potentially cause unacceptable erosion of the divertor target if the heat pulse causes the surface temperature to rise above the ablation, or melting, point of the target material. Though ELM heat flux is not usually a concern for the current generation of tokamaks, it can lead to intolerable divertor erosion when scaled to next step tokamaks. Previous scalings from low, to mid, density $\mathrm{H}$-mode operation would conclude the ablation threshold would be exceeded by a factor of 3-4 if scaled to ITER-FEAT parameters [1,3].

Several tokamaks have observed that small ELMs can be obtained at high density [3-6]. These small ELM regimes can maintain a robust pedestal pressure, yet the small energy released at each ELM would be tolerable if scaled to ITER-FEAT. However, it is unknown if these small ELM regimes will be accessible for a future large tokamak. In order to predict the target plate heat flux due to ELMs in future large devices it is important to understand which physical processes control the magnitude of the ELM heat flux. By determining which of these processes is responsible for reducing ELM energy at high density in current devices it may be possible to scale these effects to ITER-FEAT parameters.

Two general models have been put forward about the source of reduced ELM size at high density. The first model postulates that the high pedestal collisionality at high density reduces the amplitude and spatial extent of the ELM instability through a reduction in the edge bootstrap current $[7,8]$. In this model essentially all of the energy carried across the separatrix into the SOL is quickly lost through parallel transport to the target. The second model proposes that parallel transport in the SOL is the limiting process for ELM heat flux. In this scenario the ELM instability flattens the profiles across the separatrix, but the energy is limited by the sheath at the target [9]. It is not until ions traveling at the sound speed from the pedestal arrive at the target that significant ELM energy can be carried through the sheath. At high pedestal density, and low temperature, the travel time for ions becomes longer than the ELM duration resulting in a lower ELM energy. 
In this paper we examine data from DIII-D that may illuminate whether either of these scenarios, or a combination of them, is responsible for observed ELM characteristics. Data will be presented showing the scaling of energy lost from the pedestal and divertor plasma response to that energy, with the aim of providing insight into the important ELM transport processes. 


\section{ELM LOSS FROM PEDESTAL}

A series of discharges in a low triangularity, $\delta \approx 0$, lower single-null, LSN, configuration were used for this study. A shot-to-shot variation in gas puffing was used to obtain a density scan for variations in plasma current of 0.8-2.0 MA and toroidal field of 1.4-2.1 T. A few discharges were carried out at higher upper triangularity, $\delta \approx 0.4$, to assess triangularity dependence. Divertor pumping allowed for a range in the pedestal density of $2-13 \times 10^{19} \mathrm{~m}^{-3}$ corresponding to a normalized density variation of $0.2-0.9$ times the Greenwald density, $\mathrm{n}_{\mathrm{GW}}\left(10^{20} \mathrm{~m}^{-3}\right)=$ $\mathrm{I}_{\mathrm{p}}(\mathrm{MA}) /\left[\pi \mathrm{a}^{2}(\mathrm{~m})\right]$.

The primary tool for study of changes in the pedestal due to ELMs in DIII-D is the Thomson scattering diagnostic. Thomson scattering produces a very fast measurement of $\mathrm{n}_{\mathrm{e}}$ and $\mathrm{T}_{\mathrm{e}}$ at a single point in time. The DIII-D system takes 80 such measurements each second, or approximately once every $12 \mathrm{~ms}$. In order to obtain higher time resolution for profile changes across an ELM, data is collected over a period of $0.5-1.0 \mathrm{~s}$ of steady conditions with regular repeating ELMs. The data is then ordered in time to the nearest ELM in order to produce a density and temperature profile across an ELM. The pre- and post-ELM data are separately fit with a linear function as shown in Fig. 1. The value of the fit at $t=0$ is used as the pre- and post-ELM value of the density, and temperature, at that location. An example of combining the pre- and post-ELM
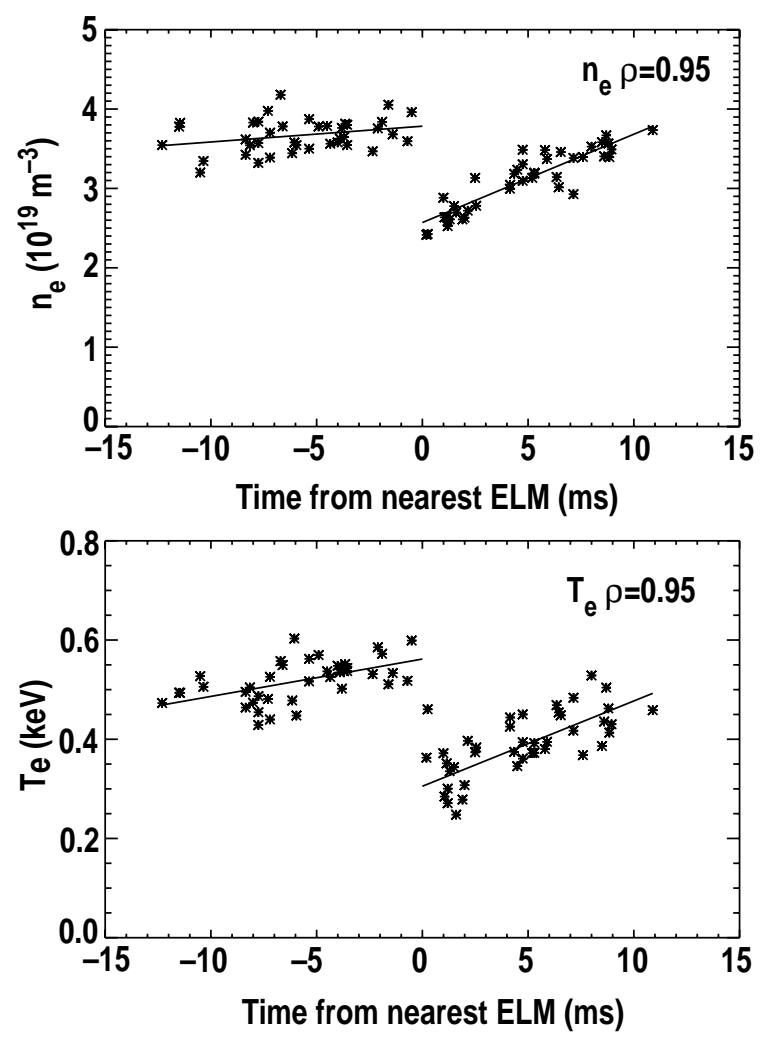

Fig. 1. Fitting of Thomson measurements at $\rho=0.95$ with respect to the nearest ELM. Both (a) density and (b) temperature are fit. values at each measurement location into a profile is shown in Fig. 2.

Clear changes are seen in the electron density and temperature profiles for this typical case. The relative perturbations to the profiles, shown in Fig. 3, are seen to extend from the separatrix into $\rho \approx 0.8$. A significant aspect of the perturbation profiles are that they are significantly wider than the steep gradient region of the pressure pedestal. Recent theoretical work $[7,8]$, postulates that the ELM instability results from a coupled peeling-ballooning mode driven by both the edge 
pressure and current gradients. The predicted eigenmodes of this instability are also much wider than the steep gradient region and are qualitatively of a similar shape to the profiles of Fig. 3 .

In order to study ELM transport processes the lost energy represented by the perturbed profiles of Fig. 2 is split into convected and conducted energy. The convected energy, $\left\langle\mathrm{T}_{\mathrm{e}}\right\rangle \Delta \mathrm{n}_{\mathrm{e}}$, results in a perturbation to the density profile and represents plasma that is convected out of the main plasma into the SOL and divertor. Changes to the temperature profile represent conduction, $<\mathrm{n}_{\mathrm{e}}>\Delta \mathrm{T}_{\mathrm{e}}$, a loss of heat from the plasma inside the separatrix.

For the ion energy convected at each ELM charge neutrality and an assumption of $Z_{\text {eff }} \sim 2.3$, typical for the discharges in this study, results in an ion convected ELM energy of $\sim 75 \%$ of that of the electrons. A more systematic measurement of the $\mathrm{Z}_{\mathrm{eff}}$ profile would be expected to produce a lower $\mathrm{Z}_{\text {eff }}$ and somewhat higher ion ELM convected energy at high density.

For the case of conducted ion ELM energy the ion temperature perturbation might be expected to be smaller than the $\mathrm{T}_{\mathrm{e}}$ perturbation because classical parallel ion thermal conductivity is smaller than the electron conductivity by a factor of $\left(\mathrm{m}_{\mathrm{e}} / \mathrm{m}_{\mathrm{I}}\right)^{1 / 2}$. To check this assumption fast, $0.5 \mathrm{~ms}$, charge-exchange recombination (CER) measurements of the ion temperature profile were made for several discharges and compared to the ELM perturbation of
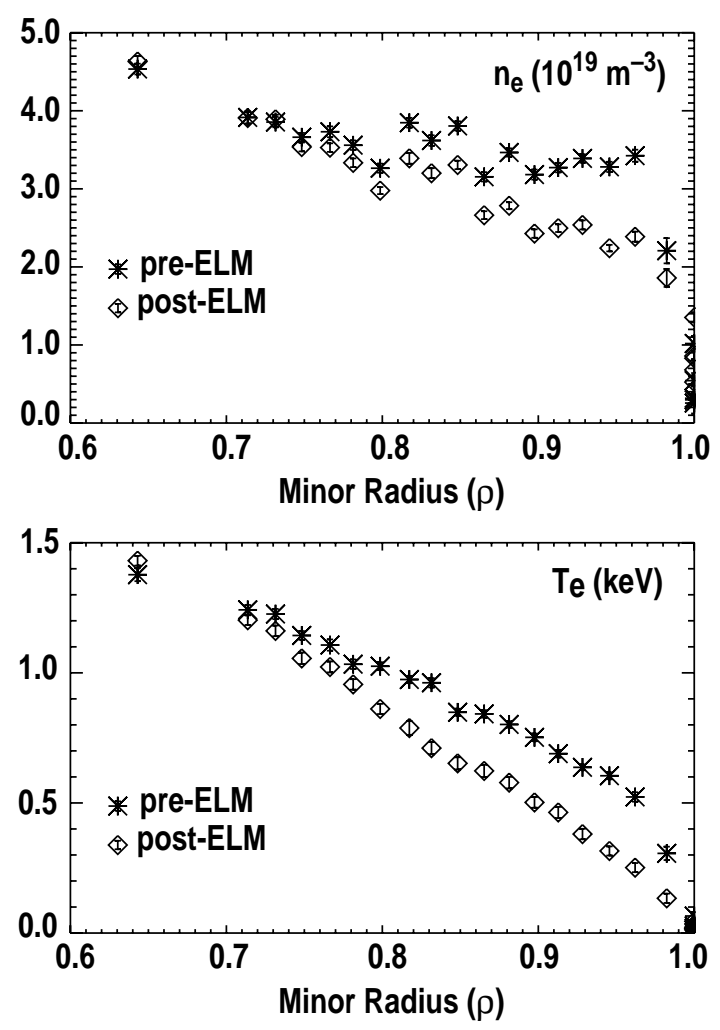

Fig. 2. Profiles of $\mathrm{n}_{\mathrm{e}}$ and $\mathrm{T}_{\mathrm{e}}$ as fit to before and after an ELM.
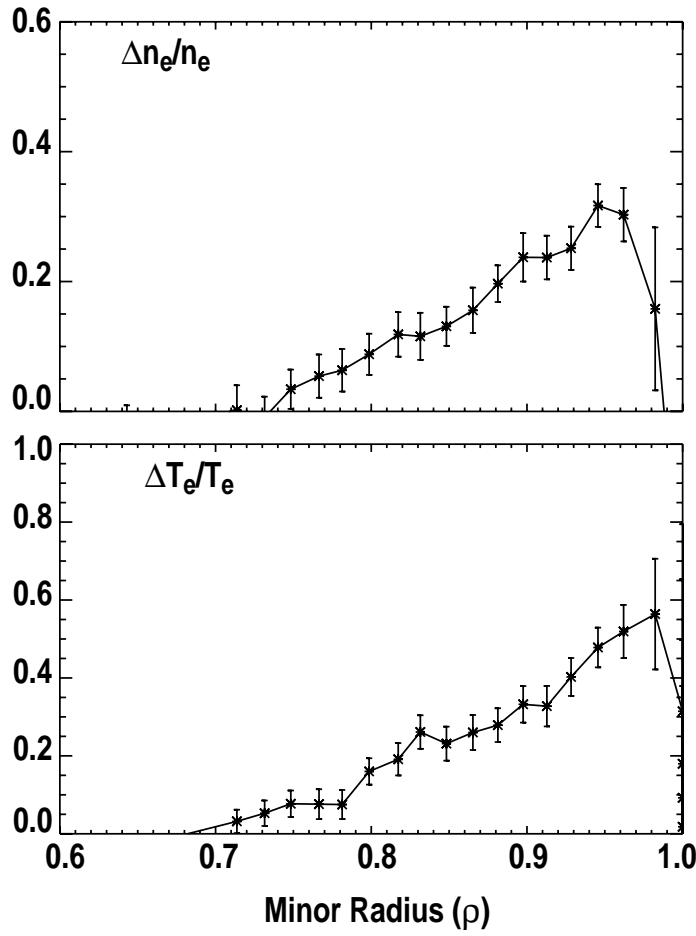

Fig. 3. Relative changes to the $\mathrm{n}_{\mathrm{e}}$ and $\mathrm{T}_{\mathrm{e}}$ profiles due to an ELM. The relative perturbation is defined and the difference between the pre- and post-ELM profiles divided by the pre-ELM profile. 
the $\mathrm{T}_{\mathrm{e}}$ profile. The ELM perturbation to $\mathrm{T}_{\mathrm{i}}$ is seen to be roughly that of the $\mathrm{T}_{\mathrm{e}}$ profile perturbation for the cases studied. Further work will be required to understand and specify the relationship between the $\mathrm{T}_{\mathrm{i}}$ and $\mathrm{T}_{\mathrm{e}}$ perturbation profiles. With the additional $\mathrm{Z}_{\mathrm{eff}}$ approximation described above it will be assumed for this study that the ions contribute an additional $75 \%$, to the ELM conducted energy as measured in the $\mathrm{T}_{\mathrm{e}}$ profiles.

For part of the data set, the ELM energy calculated from the Thomson data with the above assumptions is compared in Fig. 4 with ELM energy calculated from fast magnetic equilibrium reconstruction. Though there is significant scatter in the data, the reasonable correlation of these two independent methods suggests credibility of the Thomson profile analysis technique. A large part of the error bars shown in Fig. 4 is likely due to significant ELM-to-ELM variability. Though such variability will be important in assessing the potential for divertor ablation, this study will

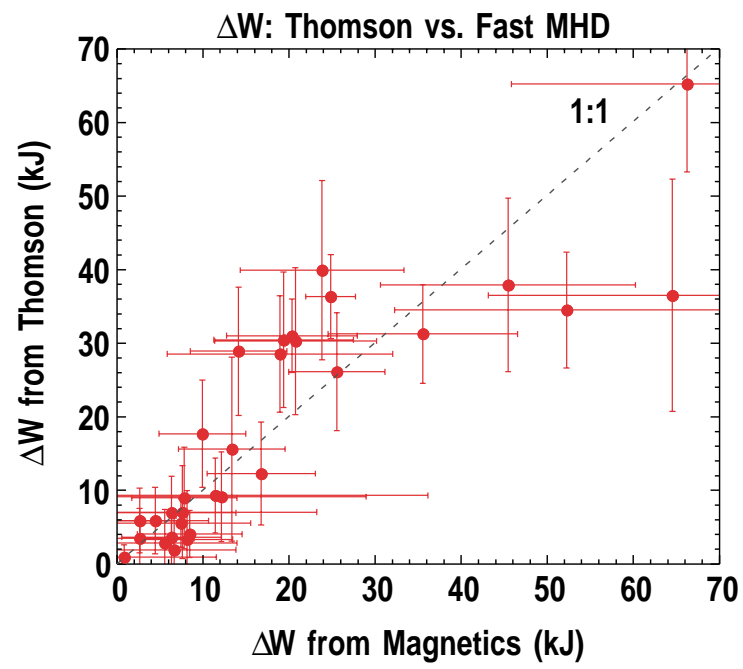

Fig. 4. ELM energy as measured by changes to the Thomson $\mathrm{n}_{\mathrm{e}}$ and $\mathrm{T}_{\mathrm{e}}$ profiles versus ELM energy as determined by fast magnetic equilibrium analysis. only consider average ELM characteristics.

The fitted Thomson pre- and post-ELM profiles were integrated separately for convected and conducted energy over the entire data set. The ELM energy lost due to convection is normalized to the pedestal energy and is plotted in Fig. 5(a) versus the pedestal Greenwald parameter, $\mathrm{n}_{\mathrm{e}, \mathrm{ped}} / \mathrm{n}_{\mathrm{GW}}$. The conducted ELM energy over the same data set is plotted in Fig. 5(b). The pedestal energy is calculated as twice the pedestal electron pressure times the plasma volume, or $2 \times 3 / 2 \times \mathrm{P}_{\mathrm{e} \text {,ped }} \times \mathrm{Vol}$. This normalization of the ELM energy has been successfully used for comparison of ELM size between different configurations and different tokamaks [1,2]. Four different cases are presented; $q_{95} \sim 3.9$ at $1.2 \mathrm{MA}$ and $2.1 \mathrm{~T}, q_{95} \sim 3.1$ at $1.2 \mathrm{MA}$ and $1.5 \mathrm{~T}, q_{95}$ $\sim 2.5$ at 2.0 MA and $2.1 \mathrm{~T}$ and high upper triangularity, $\delta \sim 0.4(\delta \sim 0$ in the other cases $)$ at 1.2 MA and 2.1 T.

Several important trends can be observed in the data of Fig. 5. First, though there is significant scatter in the data there is no obvious change in the relative convected ELM energy from low density up to $\mathrm{n}_{\mathrm{e}, \mathrm{ped}} / \mathrm{n}_{\mathrm{GW}} \sim 0.65$ where the ELMs become smaller. There is also no obvious $q 95$ dependence among the different cases. It should also be noted that the higher current and higher triangularity cases have about a factor of 2 higher pressure pedestal, but the ELM energy remains a constant fraction of the pedestal energy. 
The conducted ELM energy, however, shows a clear trend with density. The conducted ELM energy is a maximum at low density and decreases with density to near zero at $\mathrm{n}_{\mathrm{e}, \mathrm{ped}} / \mathrm{n}_{\mathrm{GW}} \sim 0.7$. At high density the scatter is due in part to the degradation in pedestal pressure at high density. This results in small ELMs near the measurement sensitivity and a large scatter in the relative ELM size. All four cases follow the same trend within the data scatter. This implies a similar density dependence for the conducted energy regardless of $q 95$, plasma current, triangularity or the pedestal pressure.

The decreasing conducted energy in Fig. 5(a) can be qualitatively explained by an edge stability model. The trends observed above in Fig. 5 are not unexpected from an edge stability point of view. The ELM instability, modeled as a peeling-ballooning mode, is driven by the steep pressure gradient in the pedestal, and the strong gradient in the pedestal bootstrap current that arises from the same pressure gradient [10]. This instability has been modeled by the ELITE code, and has produced eigenmodes for the instability that are qualitatively similar to the perturbation profiles of Fig. 3 [8]. Within this model higher density increases the edge collisionality resulting in a lower edge bootstrap current driving the instability. With lower edge current the instability is found to have a higher mode number with the eigenmodes not extending as far towards the plasma center. This is one possible factor leading to smaller ELMs at high density. Consistent with this, a factor of $\geq$

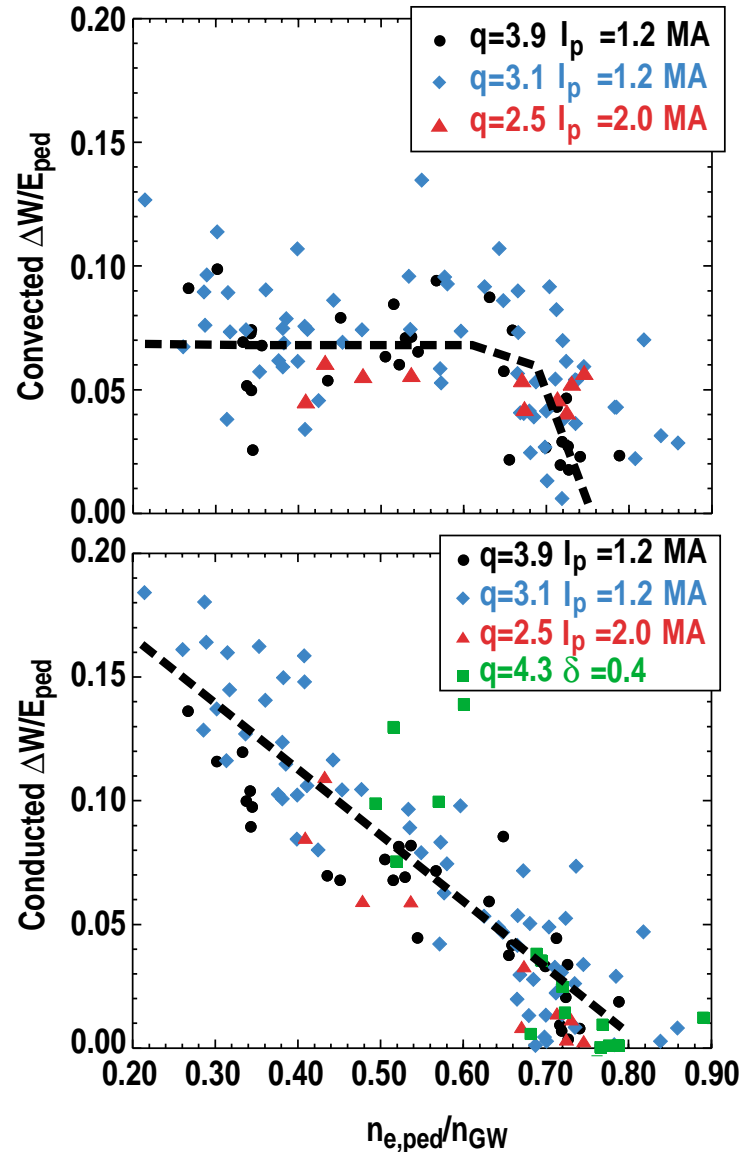

Fig. 5. (a) The normalized convected ELM energy as measured by the Thomson profile versus the pedestal density normalized by the Greenwald parameter, $\mathrm{n}_{\mathrm{e}, \mathrm{ped}} / \mathrm{n}_{\mathrm{GW}}$. (b) The normalized conducted ELM energy versus the normalized pedestal density. 5 reduction in magnetic fluctuations is measured by edge Mirnov probes at high density and an increase in the measured mode number of density fluctuations is also seen [11]. These measurements indicate a reduced ELM amplitude and/or an increase in mode number at high density.

Given this model of edge stability the ELM conducted energy data is plotted in Fig. 6 versus the pedestal electron neoclassical collisionality. This scaling tends to separate the data at small ELM energy more than the normalized density scaling of Fig. 5. In particular the high plasma 
current and high triangularity data have a higher pedestal pressure leading to a lower collisionality at the same normalized density. In addition the data set shows no consistent trend with $q$ that might be expected from the stability model. Finally the peeling-ballooning model makes no prediction concerning the relative levels of convective and conductive transport due to the instability.

There is much further work required to assess how the MHD stability affects the observed ELM heat flux. A careful comparison

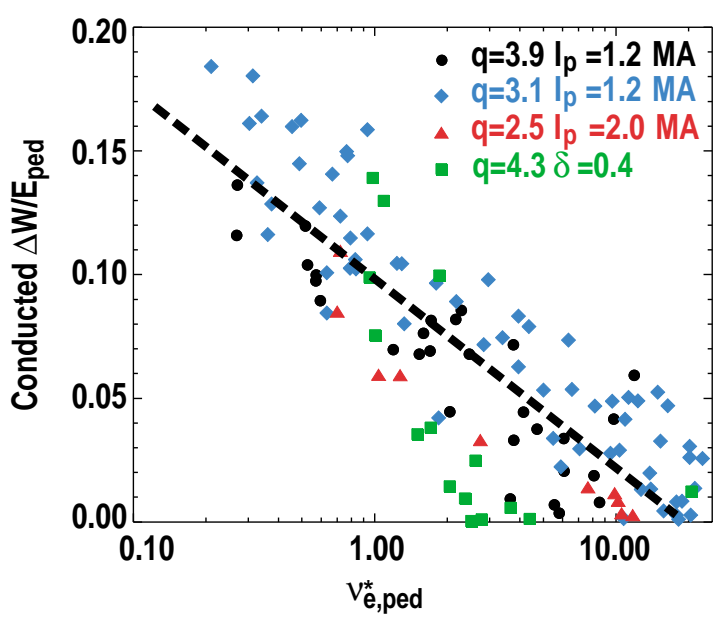

Fig. 6. The normalized conducted ELM energy versus the pedestal electron collisionality.

of the ELM energy data with stability calculations from the ELITE code using the measured pedestal will be necessary to assess the stability model. In addition the model only describes the modes at the time of onset. A description of the nonlinear evolution of the ELM will eventually be necessary to explain the observed ELM radial fluxes. 


\section{SOL AND DIVERTOR RESPONSE TO ELM ENERGY AND PARTICLES}

Though edge stability is likely necessary for understanding scaling of ELMs, SOL and divertor transport may also play an important role in determining the magnitude of energy and particles lost from the main plasma and particularly how they arrive at the divertor target. If the ELM MHD instability rapidly flattens the pressure gradient across the separatrix into the SOL, then the level of energy loss will be determined by the parallel transport time from the midplane SOL into the divertor target compared to the duration of the ELM instability.

From the SOL the fastest channel for ELM energy transport to the target plate is through electron conduction. Even for hot collisionless electrons released from the pedestal the conduction time will be very short, on the order of a few microseconds. However after a few of the fast electrons have struck the divertor target a large sheath potential is expected to develop which can be up to several times the pedestal electron temperature. This sheath potential will retard the loss of electrons and the ELM heat flux will be limited by the ion flux into the sheath. The flux into the sheath can increase when ion flux released by the ELM and traveling at the ion sound speed reaches the divertor. It is the relationship between the electron and ion travel times and the ELM duration, along with ion flux in the divertor before and during the ELM that will determine the divertor limit to ELM energy.

To study the SOL and divertor response to an ELM perturbation a number of fast diagnostics were set up in DIII-D as shown in Fig. 7. A density scan was carried out in this configuration with plasma current $1.5 \mathrm{MA}$, toroidal field $1.8 \mathrm{~T}, q_{95} \approx 3.5$ with a lower and upper triangularity of $\delta_{\mathrm{L}} \approx 0.5$ and $\delta_{\mathrm{U}} \approx 0.2$ respectively. Divertor pumping allowed a pedestal density range of 5$11 \times 10^{19} \mathrm{~m}^{-3}$, or $0.4-0.85 \mathrm{n}_{\mathrm{e}, \mathrm{ped}} / \mathrm{n}_{\mathrm{GW}}$.

The time traces of a number of diagnostics for a low density discharge are shown on an expanded scale in Fig. 8. The first trace is an edge SXR chord that mainly views the pedestal and is very sensitive to the pedestal temperature. This signal shows a fast drop in $\mathrm{T}_{\mathrm{e} \text {,ped }}$ on the order of $100 \mu \mathrm{s}$. This signal should be correlated with the conducted ELM energy described in Section 2. The drop in SXR signal is also of duration equal to, or less than, the ELM magnetic fluctuations measured by magnetic pick-up coils. At the time of the drop in the SXR signal there is a sharp rise in the divertor $\mathrm{D}_{\alpha}$ signal with no significant difference in time, $\leq 50 \mu \mathrm{s}$, for the start of this rise in the inner and outer divertors. Coincident with the rise in divertor $\mathrm{D}_{\alpha}$ is an increase in the divertor density as measured by a fast $\mathrm{CO}_{2}$ interferometer viewing vertically through the outboard divertor. The line integral of the density along this path rises from a value of $1.4 \times 10^{19} \mathrm{~m}^{-2}$ before the ELM to a peak value of $\geq 1.8 \times 10^{20} \mathrm{~m}^{-2}$ within $\approx 100 \mu$ s during an 
ELM. Much of this density decays quickly in about $100 \mu \mathrm{s}$, with the rest of the increase in density decaying on a slower timescale similar to the outer divertor $D_{\alpha}$ signal. The rise in divertor density at an ELM is estimated to typically be at least $2 \times 10^{20} \mathrm{~m}^{-3}$ given an interferometer chord path length through the divertor of $\sim 18 \mathrm{~cm}$ and the assumption that the density does not rise in the main plasma during an ELM. Before an ELM the divertor density is $\sim 4 \times 10^{19} \mathrm{~m}^{-2}$ as measured by Thomson scattering. Unfortunately the fast, but periodic Thomson diagnostic did not catch the divertor density during an ELM to confirm the interferometer measurement. The rise in density measured by the interferometer is significantly above the unperturbed divertor density and a factor of 4 above the pedestal density where the ELM originates. The implications of this large density for ELM heat flux will be discussed in the next section.

Ultimately the result of the ELM pertur-

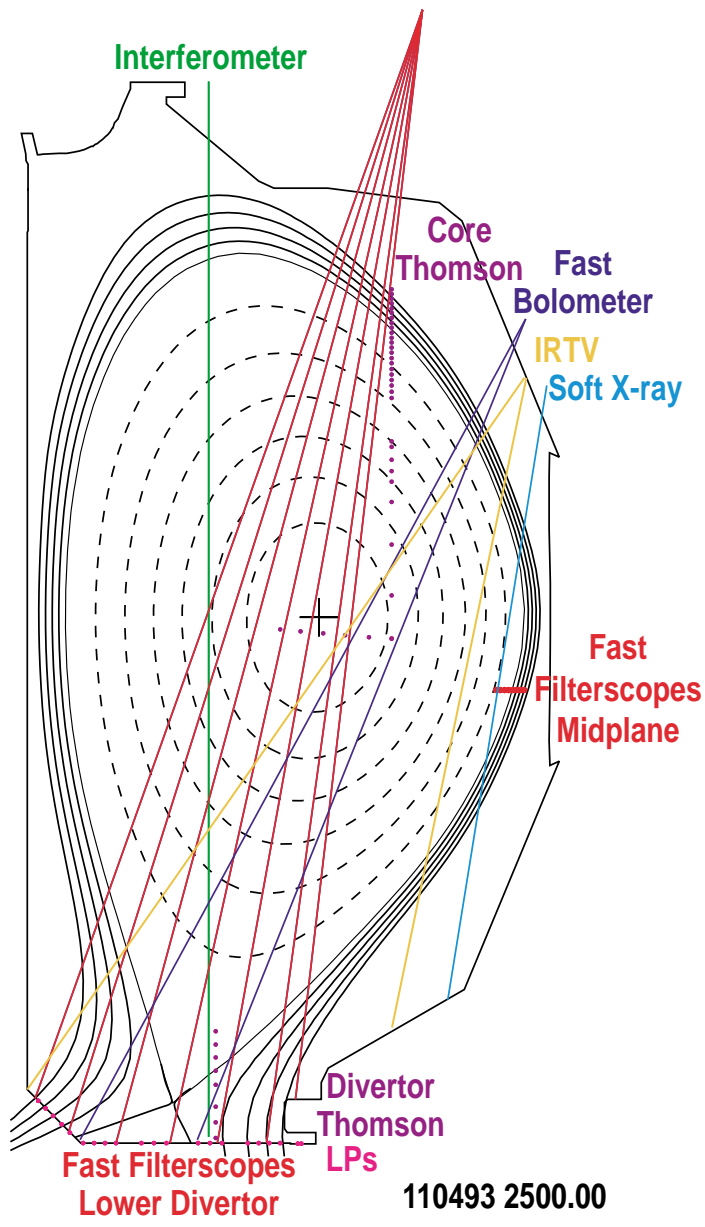

Fig. 7. The plasma configuration and extended set of pedestal and divertor diagnostics used to measure ELM perturbations.

bation is power delivered to the divertor target, also seen in Fig. 8. The rise time of the divertor power is similar to the divertor density rise with most of the power deposited in $\sim 200 \mu \mathrm{s}$, with the outboard ELM heat flux somewhat larger than the inboard. Unfortunately, a calibration uncertainty with the IR camera measuring the target plate heat temperature does not allow a determination of the absolute value of the heat flux. Past measurements have indicated that $>50 \%$ of the ELM energy lost from the pedestal is deposited on the divertor target plates. The heat flux can be integrated in time across an ELM at each radial location to obtain a radial profile of the ELM deposited energy. A profile averaged over a number of ELMs is shown in Fig. 9. The wider peak at the outboard divertor is likely due to a greater poloidal flux expansion at the target. Also shown in Fig. 8 is the radiated power coming from the inboard and outboards divertors as measured by as measured by a XUV solid-state bolometer array [12]. The in/out ratio, as well as the time dependence, of the ELM radiated power is similar to that of the target heat flux. Radiated power in regions other than the divertor is measured to be negligible in comparison to the ELM energy. 
At high density a different ELM behavior is observed as shown in Fig. 10. In this case gas puffing raises the pedestal density to $\approx$ $1.0 \times 10^{20} \mathrm{~m}^{-3}$, or $0.80 \times \mathrm{n}_{\mathrm{e}, \mathrm{ped}} / \mathrm{n}_{\mathrm{GW}}$. First the ELM perturbation to the edge SXR signal is much smaller than at low density. This is consistent with the earlier pedestal profile data of Section 2 where little or no perturbation to the temperature profile was observed at high density. There is evidence of particle loss in the SOL as the midplane $D_{\alpha}$ rises at each ELM. However, the outboard divertor is quite different at high density. Between ELMs the outboard divertor is in a detached condition with $\mathrm{n}_{\mathrm{e}} \approx 4 \times 10^{20} \mathrm{~m}^{-3}$ and $\mathrm{T}_{\mathrm{e}}<5 \mathrm{eV}$ as measured by the divertor Thomson diagnostic. At an ELM the divertor density drops as evidenced by both the outboard divertor $D_{\alpha}$ and a decrease in the divertor interferometer signal. This drop occurs over about $200 \mu$ s and is an indication of the divertor plasma reattaching due to the additional ELM energy flux. Divertor Thomson measurements during the low density phase of the ELM are consistent with the fast interferometer signal and indicate a lower density, $\mathrm{n}_{\mathrm{e}} \approx 3 \times 10^{19} \mathrm{~m}^{-3}$ and a higher temperature, $\mathrm{T}_{\mathrm{e}}>20 \mathrm{eV}$, during this phase. After about $2 \mathrm{~ms}$ the energy of the ELM is dissipated and the outboard divertor again detaches. The divertor heat flux is now less at high density, as would be expected from

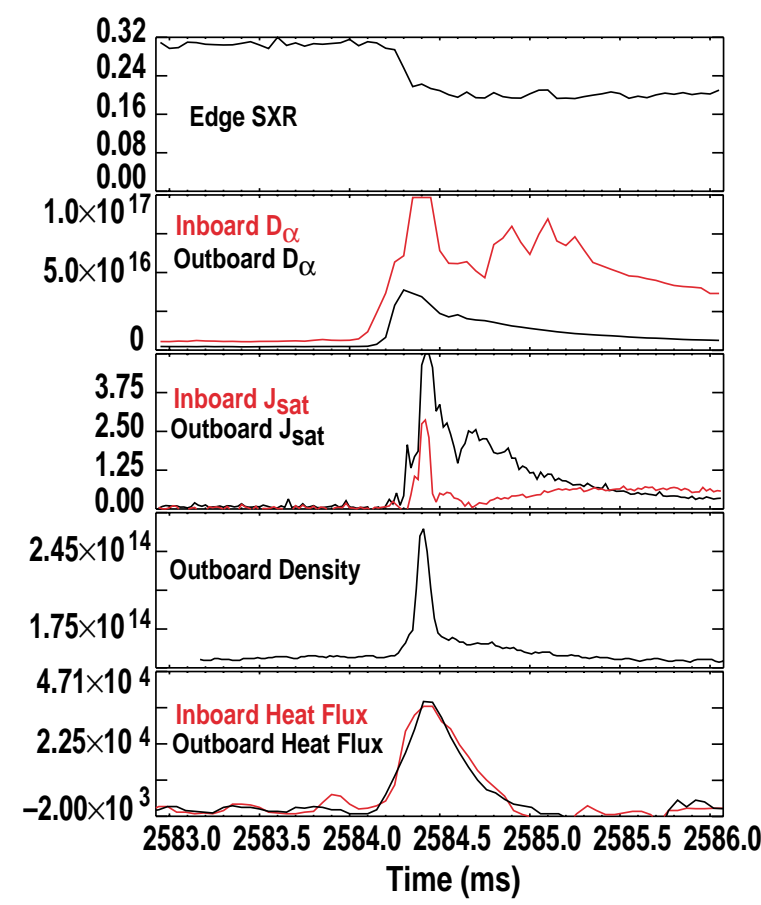

Fig. 8. Time behavior of ELM characteristics at low density. Shown are the pedestal SXR, inboard and outboard divertor $\mathrm{D}_{\alpha}$, inboard and outboard $\mathrm{J}_{\text {sat }}$ from Langmuir probes, the interferometer signal passing through outboard divertor, and the peak heat flux at the inboard and outboard divertor.

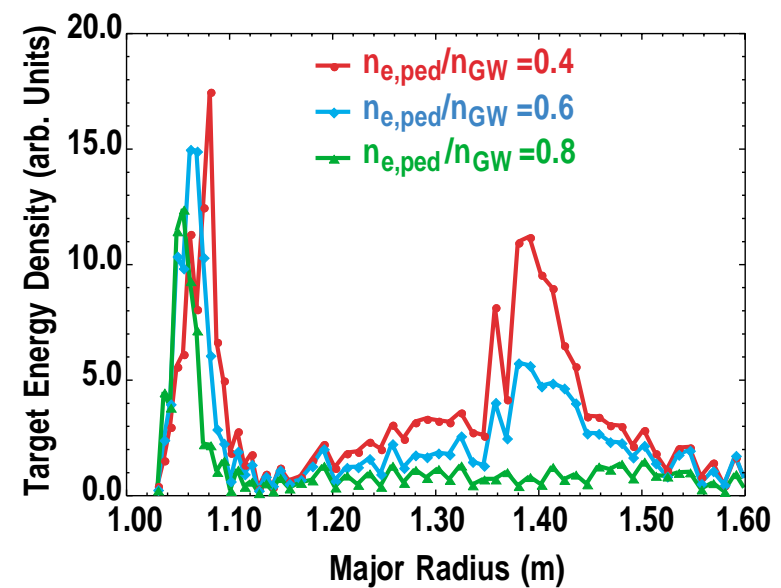

Fig. 9. The radial profile of the ELM deposited energy on the divertor target. Shown are profiles for pedestal densities of $0.4,0.6$ and 0.8 of the Greenwald density limit.

the earlier observed smaller pedestal energy drop. The profile of ELM energy deposited on the target at intermediate and high density is shown in Fig. 9. As density increases and the ELM energy decreases the biggest change to the divertor heat flux is to the outboard divertor. The fast radiated power measurements also reflect the in/out asymmetry observed with the IR camera. The implications of these trends will be discussed in the next section. 


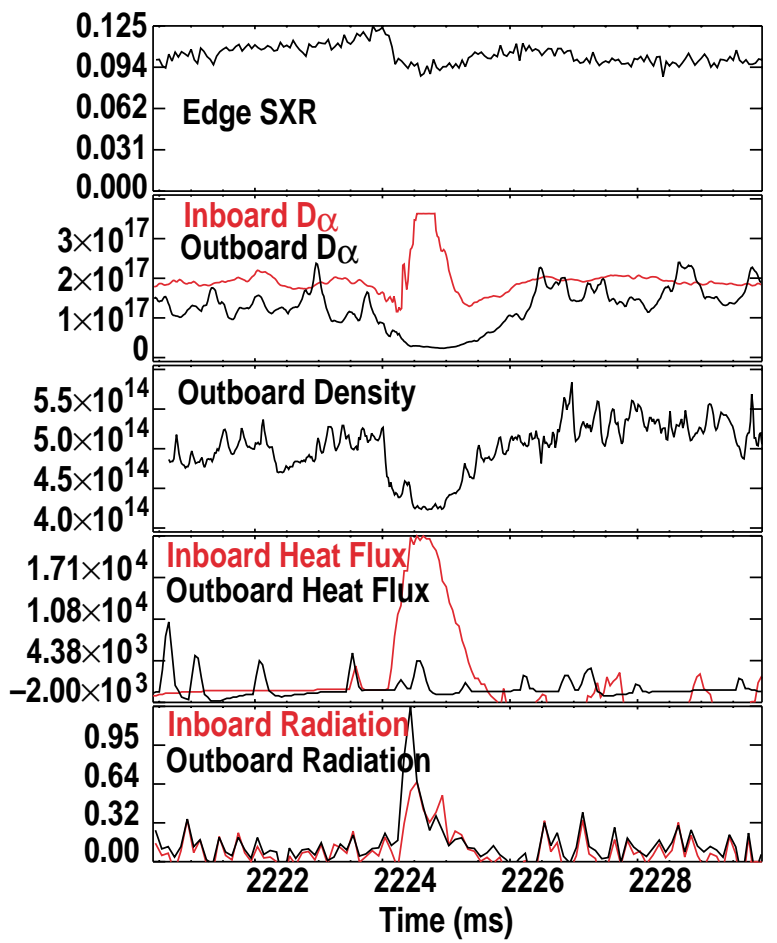

Fig. 10. Time behavior of ELM characteristics at low density. Shown are the pedestal SXR, inboard and outboard divertor $\mathrm{D}_{\alpha}$, the interferometer signal passing through outboard divertor, the inboard and outboard peak divertor heat flux and the radiation at the inboard and outboard divertor. 


\section{DISCUSSION}

To interpret the divertor data and understand its role in setting the divertor ELM heat flux we first examine parallel transport of electron and ion energy in the SOL. The perpendicular ELM flux into the SOL has been observed to occur at the outer midplane [13-15]. For this data set the parallel path length from the outer midplane is $12 \mathrm{~m}$ to the outer divertor and $30 \mathrm{~m}$ to the inner divertor. With a pedestal temperature of $750 \mathrm{eV}$ at low density, hot electrons from the pedestal can travel to either target in less than $10 \mu \mathrm{s}$, much shorter than ELM instability or ELM heat flux duration. However, the loss of fast electrons, and the ELM heat flux, will be limited by a build up of the sheath potential to maintain charge neutrality. In this condition the heat flux will be set by the local ion flux at the sheath. The local ion flux, and thus the ELM heat flux, can increase with the flux of ions from the pedestal traveling at the ion sound speed. For the low density case this is a parallel time of $\tau_{\| \text {,out }} \sim 50 \mu$ s to the outer divertor and $\tau_{\| \text {,in }} \sim 115 \mu$ s to the inner divertor. This parallel transport time for ions is of the same order as the ELM instability duration as seen by magnetic fluctuations and the drop in the pedestal SXR signal. This similarity in timescales might account for the observed density dependence for the conducted energy. At low density and high pedestal temperature ion flux from the pedestal reaches the target before the ELM instability has ended. This allows additional power to be conducted from the midplane and pedestal. At higher pedestal densities, and lower temperatures, the parallel ion transport time increases and does not reach the sheath before the end of the ELM instability. In this case the ELM energy will be limited by the convection of particles from the pedestal into the SOL.

The fast rise in density observed by the $\mathrm{CO}_{2}$ interferometer appears to contradict this scenario. With other interferometer chords passing through the main plasma, but not the divertor, showing a drop in density at the ELM, the rise on this chord should be attributed to a fast rise in the divertor density. The increase in divertor density of $2 \times 10^{20} \mathrm{~m}^{-3}$ is at least a factor of 4 greater than the pedestal density. One possible cause of the divertor density increase is the release of neutrals from the target. Fast electrons from the pedestal could build the target sheath to $1 \mathrm{kV}$ or more. A local ion falling through this sheath would gain enough energy to dislodge several neutrals in the saturated target. Greater than unity recycling could quickly generate sufficient ion flux to allow fast conduction of all electron energy crossing the separatrix into the SOL. The conducted ELM energy would then be set by the midplane ELM MHD instability.

Another contradictory observation is the in/out asymmetry in the ELM divertor heat flux. At low density the shorter ion transport time to the outer divertor should result in a greater ion flux and resulting heat flux. A diagnostic issue associated with interpreting IR measurements from 
different surface properties at the inboard and outboard divertor targets has been given as possible explanation for the observed ELM heat flux asymmetries [1,16,17]. However, the data presented here shows the asymmetry to become larger at high density. This suggests some property other than ion transport time, such as neutrals available for ionization, is more important for determining the ELM heat flux.

At this time it is not possible to unambiguously determine if the observed scaling of ELM divertor heat flux is being set by the MHD instability of the pedestal or parallel transport processes in the divertor. Modeling of the SOL transport will be a challenging task requiring coupling of kinetic effects, populations of fast electrons and ions in a background plasma, with materials properties such as recycling and impurity generation. Some efforts in this direction are now underway [18]. Predicting MHD transport will also be a difficult task involving nonlinear evolution of the unstable modes. These processes must be looked at together to predict ELM characteristics in a future device such as ITER-FEAT. If these different processes scale differently then a parameter controlling ELM heat flux in today's devices might not be the limiting factor for ITER-FEAT.

A high priority should also be placed on exploring regimes that produce small ELMs at low pedestal collisionality. Today's devices achieve small ELMs at high density, but the pedestal collisionality is also low. To obtain adequate confinement ITER-FEAT will require a pedestal at low collisionality, even at a high Greenwald density. Small ELM regimes must be found that can scale to larger tokamaks. 


\section{REFERENCES}

[1] A.W. Leonard, A. Herrmann, K. Itami, et al., J. Nucl. Mater. 266-269 (1999) 109.

[2] G. Janeschitz, et al., J. Nucl. Mater. 290-293 (2001) 1.

[3] A. Loarte, M. Becoulet, G. Saibene, et al., accepted for publication in Plasma Phys. and Cont. Fusion

[4] A.W. Leonard, T. H. Osborne, M.E. Fenstermacher, et al., J. Nucl. Mater. 290-293 (2001) 1097.

[5] J. Stober, M. Maraschek, G. D. Conway, et al., Nucl Fusion 41 (2001) 1123.

[6] G. Saibene, et al., submitted to Plasma Phys. Control. Fusion.

[7] J.W. Conner, et al., Phys. Plasmas 5 (1998) 2687.

[8] P.B. Snyder, H.R. Wilson, J.R. Ferron, et al., "Edge Localized Modes and the Pedestal: A Model Based on Coupled Peeling-Ballooning Modes," accepted for publication in Phys. Plasmas (2002).

[9] A. Loarte, et al., Fusion Energy 2000 ITERP/11(R), Proc. 18th IAEA Fusion Energy Conference (Sorrento, Italy, 2000), IAEA, Vienna 2001 (ISSN 1562-4153).

[10] O. Sauter, et al., Phys. Plasmas 6 (1999) 2834.

[11] T.H. Osborne, et al., Phys. Plasmas 8, (2001) 2017.

[12] D.S. Gray, S.C. Luckhardt, E. Hollmann, et al., Proc. 27th EPS Conf. On Controlled Fusion and Plasma Physics, Budapest, Hungary, 2000, Vol. 4121.

[13] A. Loarte, et al., Proc. 28th EPS Conf. On Controlled Fusion and Plasma Physics, Madeira, Portugal, 2001, P3.005.

[14] A. Chankin, et al., Proc. 8th IAEA Tech, Com H-mode \& Transport Barrier Physics, Toki, Japan, 2001. To be published in Plasma Phys. Control. Fusion.

[15] L. Horton, et al., Proc. 9th European Fusion Physics Workshop, Saariselka, Finland 2001.

[16] A. Herrmann, et al., Plasma Phys. Control. Fusion 37 (1995) 17.

[17] A.W. Leonard, W. Suttrop, T.H. Osborne, et al., J. Nucl. Mater. 241-243 (1997) 628.

[18] A. Bergmann, accepted for publication in Nucl. Fusion. 


\section{ACKNOWLEDGMENT}

Work supported by U.S. Department of Energy under Contracts DE-AC03-99ER54463, W-7405-ENG-48, DE-AC04-94AL85000, and Grant DE-FG03-95ER54294. 